\title{
LUMINOSITY DENSITY EVOLUTION IN THE UNIVERSE AND COSMOLOGICAL PARAMETERS
}

\author{
TOMONORI TOTANI \\ Department of Physics, The University of Tokyo \\ Tokyo 113, Japan \\ totani@utaphp2.phys.s.u-tokyo.ac.jp
}

\begin{abstract}
Star formation history in galaxies is strongly correlated to their present-day colors and the Hubble sequence can be considered as a sequence of different star formation history. Therefore we can model the cosmic star formation history based on the colors of local galaxies, and comparison to direct observations of luminosity density evolution at high redshift gives a new test for the cosmological parameters which is insensitive to merger history of galaxies. The luminosity density evolution in $0<z<1$ observed by the Canada-France Redshift Survey in three wavebands of $2800 \AA, 4400 \AA$, and $1 \mu \mathrm{m}$ indicates that the $\Lambda$-dominated flat universe with $\lambda_{0} \sim 0.8(>0.53$ at $95 \% \mathrm{CL}$ ) is strongly favored.

The cosmic star formation rate (SFR) at $z>2$ is also compared to the latest data of the Hubble Deep Field including new data which were not incorporated in the previous work of Totani, Yoshii, \& Sato (1997), and our model of the luminosity density of spiral galaxies taking account of gas infall is consistent with the observations. Starbursts in elliptical galaxies, which are expected from the galactic wind model, however overproduce SFRs and hence they should be formed at $z \gtrsim 5$ or their UV emission has to be hidden by dust extinction. The amount of metals in galactic winds and escaping ionizing photons are enough to contaminate the Ly $\alpha$ forests or to reionize the universe.
\end{abstract}

\section{Introduction}

Recently the cosmic star formation history is becoming a very hot topic mainly because direct observations of luminosity density evolution in the 
universe at high redshifts become possible. The cosmic star formation history is itself very interesting, but here we stress that it is also important for many related topics in astrophysics, e.g., relic supernova neutrinos (Totani, Sato, \& Yoshii 1996), estimate of the cosmological parameters (Totani, Yoshii, \& Sato 1997; TSY), and gamma-ray bursts brightness distribution analysis (Totani 1997). Here we describe mainly the work of TSY which showed that the marked evolution in the luminosity density of galaxies in $z<1$.observed by the Canada-France Redshift Survey (CFRS; Lilly et al. 1996) favors the existence of non-vanishing cosmological constant, $\Lambda$, in all the three wavebands of restframe $2800 \AA, 4400 \AA$, and $1 \mu \mathrm{m}$. The latest data of cosmic star formation rate at $z>1$, which were not available in TYS, are also newly incorporated here and star formation history at higher redshifts is discussed.

The present-day colors of galaxies are known to be strongly correlated with their morphological type through type-dependent history of star formation, and therefore it is possible to construct a model of cosmic star formation history by summing up type-dependent evolution models of galaxies with relative proportion of their types. If the global history of star formation for the composite of all types of galaxies is determined in this way, cosmological models can then be tested against the observations of the luminosity density evolution in the universe. We calculate an evolution of the luminosity density using the galaxy evolution models of population synthesis developed by Arimoto \& Yoshii (1987, hereafter AY) and Arimoto, Yoshii, \& Takahara (1992, hereafter AYT) where the effect of chemical evolution is appropriately taken into account. Since the population synthesis models of galaxies are based on the present-day colors of galaxies, the star formation history to a look-back time corresponding to an average age of stars in a galaxy is especially well modeled. These models are therefore reliable especially at low redshifts of $z<1$ where we constrain the cosmological parameters from the CFRS data.

\section{Luminosity Density Evolution and Cosmology}

Fig. 1 shows the luminosity density evolution as a function of time for each galaxy type calculated by the AY and AYT models. For the detail of the calculation, see TYS. The comparison to the CFRS data is performed in $z<1$, and this corresponds to $t>\mathrm{a}$ few Gyrs in the figure, where $t$ is the time elapsed from formation of galaxies. (The formation epoch of $z_{F}=5$ is assumed in this figure.) In short wavebands such as $2800 \AA$, the luminosity density is dominated by Sab and Sbc galaxies, while the long waveband of $1 \mu \mathrm{m}$ is dominated by $\mathrm{E} / \mathrm{S} 0$ galaxies. The contribution by late-type spirals or irregular galaxies is relatively small. 


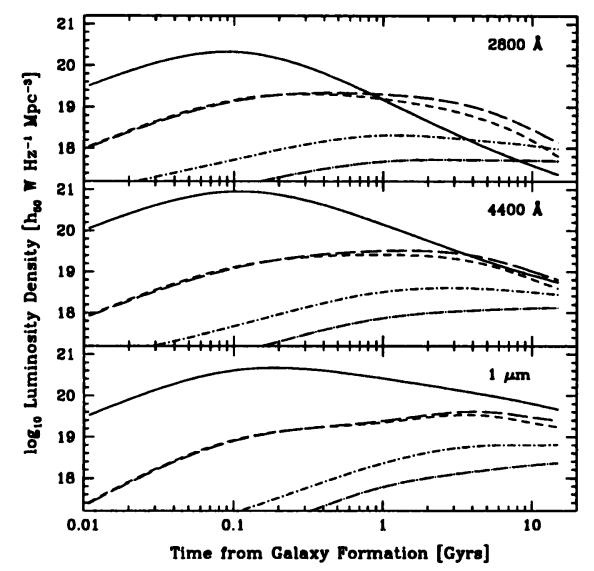

Figure 1. Evolution of the comoving luminosity density at $2800 \AA, 4400 \AA$ and $1 \mu \mathrm{m}$ as a function of time for model galaxies of different morphological types. The galactic wind model from AY is used for E/S0 (solid line), and the S1 models from AYT for spiral galaxies of Sab (short-dashed), Sbc (long-dashed), Scd (dot-short-dashed), and Sdm (dot-long-dashed). The luminosity density at 15 Gyrs for the composite of all types is normalized to $\mathcal{L}(4400 \AA)=10^{19.296} h_{50} \mathrm{~W} \mathrm{~Hz}^{-1} \mathrm{Mpc}^{-3}$.

We consider three representative cosmological models: the Einstein-de Sitter (EdS) universe with $\left(\Omega_{0}, \lambda_{0}\right)=(1,0)$, an open universe with $(0.2,0)$, and a $\Lambda$-dominated, flat universe with $(0.2,0.8)$. The redshift of galaxy formation is assumed to be $z_{F}=5$. Different values of $z_{F}$ hardly change our result as far as we restrict ourselves in $z<1$. The Hubble constant $h \equiv H_{0} /(100 \mathrm{~km} / \mathrm{s} / \mathrm{Mpc})$ is taken as $0.5,0.6$, and 0.7 for the three cosmological models in order to give a reasonable age of galaxies $(12.1,12.4$, and 13.6 Gyrs, respectively). In Fig. 2, we show the comoving luminosity densities at $2800 \AA, 4400 \AA$, and $1 \mu \mathrm{m}$ as a function of $z$ for the EdS universe (left panel), the open universe (middle panel), and the $\Lambda$-dominated universe (right panel). The CFRS data points are those from the "luminosityfunction-estimated" values (Lilly et al. 1996) for $2800 \AA$ (circles), $4400 \AA$ (triangles), and $1 \mu \mathrm{m}$ (squares). The four theoretical curves for each waveband correspond to the variation of the evolution models of spiral galaxies: S1(solid), S2(dashed), I1(dot-short-dashed), and I2(dot-long-dashed). These four models are used to estimate the possible uncertainties in star formation history of spiral galaxies (see AYT for detail). It is clear from Fig. 2 that the observed $\mathcal{L}$-evolution at $z=0-1$ becomes flatter with decreasing $\Omega_{0}$ or increasing $\lambda_{0}$, and eventually falls in agreement with the $\Lambda$-dominated universe. In sharp contrast, however, the observed $\mathcal{L}$-evolution is too steep to agree with the EdS or open universe, and such a discrepancy 


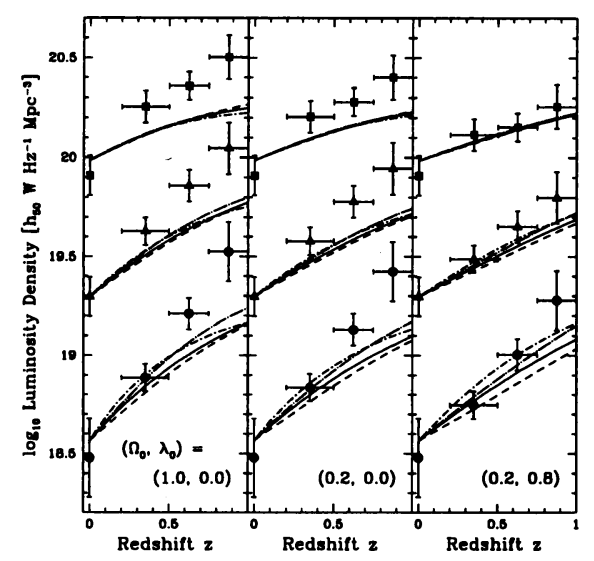

Figure 2. Evolution of the comoving luminosity density as a function of redshift for the composite of all galaxy types, shown for the Einstein-de Sitter universe with $\left(\Omega_{0}, \lambda_{0}\right)=(1,0)$ (left panel), the open universe with $(0.2,0)$ (middle panel), and the $\Lambda$-dominated flat universe with $(0.2,0.8)$ (right panel). The CFRS data points (Lilly et al. 1996), scaled to $h=0.5$, are shown by circles $(2800 \AA)$, triangles $(4400 \AA)$, and squares $(1 \mu \mathrm{m})$. The four theoretical curves for each of $2800 \AA, 4400 \AA$, and $1 \mu \mathrm{m}$ correspond to the evolution models of S1 (solid line), S2 (dashed), I1 (dot-short-dashed), and I2 (dot-long-dashed) for spiral galaxies. The galactic wind model for elliptical galaxies is used in common. The curves are normalized to coincide with the $4400 \AA$ data at $z=0$.

is much more considerable at $1 \mu \mathrm{m}$ where elliptical galaxies are dominant and uncertainty in star iormation history is very small.

For the purpose of quantitative comparison, we have calculated the slope index of the $\mathcal{L}$-evolution, $\alpha \equiv d \log \mathcal{L} / d \log (1+z)$, averaged over the range of $z=0-1$. Lilly et al. (1996) estimated the observed slope for the EdS cosmology as $\alpha=3.90 \pm 0.75(2800 \AA), 2.72 \pm 0.5(4400 \AA)$, and $2.11 \pm 0.5$ $(1 \mu \mathrm{m})$. Using these observational errors, we have performed a $\chi^{2}$ analysis for $\alpha$ and found that the EdS and open cosmologies are inconsistent with the data with $99.86 \%$ C.L. and $98.6 \%$ C.L., respectively. Assuming a flat universe $\left(\Omega_{0}+\lambda_{0}=1\right)$ with $h=0.6$, the lower limit on $\lambda_{0}$ is obtained as 0.37 (99\% C.L.) or 0.53 (95\% C.L.). Here, one of the galaxy evolution models (S1-I2) is used that gives the most conservative result in the $\chi^{2}$ analysis.

\section{Discussion}

Conventional tests on cosmological parameters based on number density of galaxies, such as galaxy number counts or gravitational lensing probability in high redshift objects, generally suffer uncertainties in merger history of 


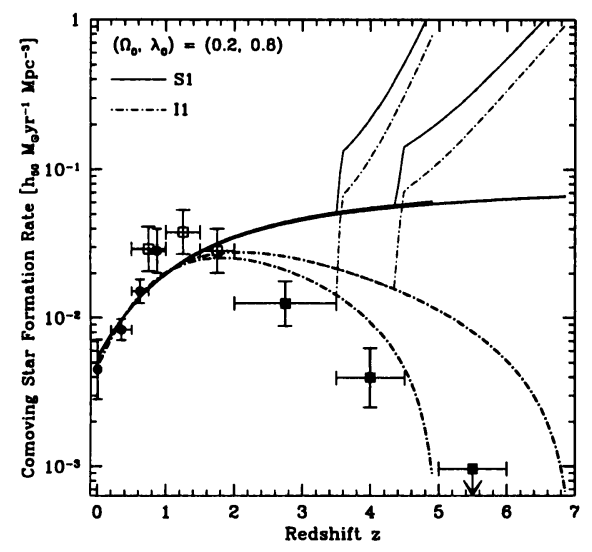

Figure 3. Star formation rate (SFR) in the universe as a function of redshift. The $\Lambda$-dominated flat universe with $\left(\Omega_{0}, \lambda_{0}\right)=(0.2,0.8)$ is assumed for both predicted and observed SFRs shown in this figure after scaled to $h=0.5$. The data points are from Lilly et al. (1996; filled circles), Madau et al. (1997; filled squares) and Connolly et al. (1997; open squares). The theoretical curves are based on the S1 (solid) or I1 (dot-dashed) models for spiral galaxies and the galactic wind model for elliptical galaxies. All galaxies are assumed to be formed at $z_{F}=5$ or 7 , with $h=0.7$. The SFRs from spiral galaxies only are shown by thick lines, while those from all types including elliptical galaxies by thin lines. The theoretical curves are normalized to agree with the data at $z<1$.

galaxies. Most publications of this kind assumed a constant comoving number density, which has not completely been confirmed by observations. In contrast, we stress that the new test presented here is based on the relation between evolutionary steepness of cosmic star formation rate and presentday colors of local galaxies, which is essentially determined by the stellar evolution theory and the initial mass function. The luminosity density is relatively insensitive to merger of galaxies, because increase in number density of galaxies is compensated by corresponding decrease in luminosity of each galaxy. Starbursts associated with mergers might lead to complicated star formation history in a galaxy, but the CFRS has shown that the luminosity density evolution is smooth at least when averaged over the universe.

The test on cosmological parameters is performed in $z<1$, where the uncertainties in both theory and observation are relatively small. Recent progress in direct observations of star-forming galaxies at $z \gtrsim 2$ however provides important information for star formation history in the universe. In Fig. 3, assuming the $\Lambda$-dominated universe with $\left(\Omega_{0}, \lambda_{0}\right)=(0.2,0.8)$, we plot the observed star formation rates (SFRs) in the universe which are compiled by Madau (1997) and Connolly et al. (1997), together with the SFRs predicted by our model with $z_{F}=5$ and 7 assuming $h=0.7$. Thick 
lines show the SFRs from spiral galaxies, and thin lines from all types including elliptical galaxies. Since the absolute SFRs are uncertain by a factor of $2-3$, we normalize the curves to agree with the data at $z<1$. Inspection of this figure clearly indicates that the luminosity density of spiral galaxies taking account of gas infall (I1 model) with $z_{F} \sim 5$ is consistent with the observational data within the observational uncertainties.

On the other hand, starbursts in elliptical galaxies, which are expected from galactic wind models, seem to overpredict the SFR density in this figure, although an unrealistic assumption of one unique formation redshift for all ellipticals exaggerates the overproduction. There are some possibilities to resolve this contradiction: 1) elliptical galaxies are formed at $z_{F} \gtrsim 5$, 2) most of UV emission from elliptical starbursts is hidden by dust, and 3) elliptical galaxies are formed without starburst phases, such as formation from mergers of smaller systems. Maoz (1997) investigated the first possibility and he found that passively evolving elliptical galaxies formed at $z \gtrsim 5$ are below the detection limits of the HDF. Meurer et al. (1997) suggest a significant UV extinction by dust for starburst galaxies at high redshifts and observation of the HDF by Infrared Space Observatory also suggests existence of starbursting ellipticals which could not be detected in restframe UV lights (Rowan-Robinson et al. 1997). Therefore the overproduction of SFRs in ellipticals is not so serious at the present stage. Finally, we point out that the galactic wind model used here ejects $7 \%$ of its baryon mass and the amount of metals ejected with this wind is sufficient to contaminate the intergalactic medium as observed in Ly $\alpha$ forests. If the escape fraction of UV emission is also as large as $7 \%$ (for typical escape fractions, see e.g., Madau \& Shull 1996), the ionizing photons are enough to reionize the universe. (see TYS for detail.)

This work has been supported in part by the Scientific Research Fund No. 3730 of the Ministry of Education, Science, and Culture of Japan.

\section{References}

Arimoto, N. \& Yoshii, Y. 1987, A\&A, 173, 23 (AY)

Arimoto, N., Yoshii, Y., \& Takahara, F. 1992, A\&A, 253, 21 (AYT)

Lilly, S. J., Fèvre, O. Le, Hammer, F., \& Crampton, D. 1996, ApJ, 460, L1

Connolly, A. J. et al. 1997, ApJ, 486, L11

Madau, P. \& Shull, J. M. 1996, ApJ, 457, 551

Madau, P. 1997, in Star Formation Near and Far, eds. S. S. Holt \& G. L. Mundy (AIP: New York), p481

Maoz, D. 1997, preprint, astro-ph/9704173 (to appear in ApJL)

Meurer, G. R. et al. 1997, AJ, 114, 54

Rowan-Robinson, M. et al. 1997, MNRAS, 289, 490

Totani, T., Sato, K. \& Yoshii, Y. 1996, ApJ, 460, 303

Totani, T., Yoshii, Y. \& Sato, K. 1997, ApJ, 483, L75 (TYS)

Totani, T. 1997, ApJ, 486, L71 